\title{
Case Report: General Anesthetic Management for Laparoscopic Cholecystectomy in Paramyotonia Congenita
}

Analise McGreal,' Daniel Slagle, ${ }^{1}$ Andrew Dickens. ${ }^{2}$

\begin{abstract}
Background: Paramyotonia congenita (PC) is a rare disorder affecting skeletal muscle. Patients with this non-progressive condition experience intermittent episodes of sustained myotonia. Due to the predisposition for prolonged muscle contraction, special attention must be given to anesthetic management during operative procedures to prevent complications similar to those experienced in patients with malignant hyperthermia. To date, however, limited reports of anesthetic management in paramyotonia congenita are available. The Case: The present report describes successful general anesthetic management given to a patient with paramyotonia congenita using propofol and ketamine for induction, non-depolarizing rocuronium for muscle paralysis, and continuous nitrous oxide and IV propofol infusion for sedation. The patient remained stable throughout the case without myotonic episodes or other complications. Conclusion: Our report describes successful anesthetic management in a patient with paramyotonia congenita. This provides a potential management plan that may be applied to PC patients undergoing a variety of surgical procedures and eliminates risk associated with succinylcholine and possibly volatile anesthetics. Further research is needed to determine whether this approach is superior to previously reported techniques and should also identify which agents may be effectively utilized to reverse an intra-operative myotonic episode in PC.
\end{abstract}

Key Words: Anesthesia; Paramyotonia congenita; Laparoscopic cholecystectomy; Sodium channel; Ceneral anesthesia; Malignant hyperthermia (Source: MeSH-NLM).

\begin{abstract}
Introduction
Paramyotonia congenita (PC) is a rare muscular disorder with an estimated prevalence between $1 / 100,000$ to $1 / 200,000 .^{1}$ The disorder is characterized by periodic muscle contraction, or myotonia, followed by flaccid paralysis. ${ }^{1}$ It is caused by an autosomal-dominant mutation in the SCN4A gene, which is normally responsible for regulating the amount of sodium and potassium ions present across the skeletal muscle cell membrane. Therefore, the mutation in this gene is thought to interfere directly with normal muscle contraction and relaxation. ${ }^{1}$ Most patients with PC experience periodic myotonic contraction and paralysis by adolescence. However, in contrast to some other muscular disorders, PC is non-progressive and patients are expected to have a normal life expectancy.' Many patients with PC do not require pharmacologic intervention and treatment is primarily symptomatic. For example, symptomatic treatment may include reduction of cold exposure, which may act as a trigger for myotonic episodes. ${ }^{1}$ Patients who do receive pharmacologic treatment may be treated with mexiletine for muscle relaxation.
\end{abstract}

Patients with PC experience a broad range of symptom severity. They are at increased risk when undergoing general anesthetic management, especially with succinylcholine (Figure 1). ${ }^{1-2}$ While this is similar to the better-known presentation of malignant hyperthermia (MH), it is important to note that patients with PC are not at an increased risk for malignant hyperthermia. ${ }^{3}$

MH is usually triggered by succinylcholine and/or halogenated volatile anesthetics and presents with hypercarbia, muscle rigidity, hyperthermia, and rhabdomyolysis following anesthetic induction. ${ }^{3}$ In patients with $\mathrm{PC}$, the only symptom is myotonia, typically in the hands, face, or neck. ${ }^{4}$ Nevertheless, there is clear risk for patients with PC who

\section{Highlights:}

- In this case report, we describe general anesthetic induction and management for a patient with paramyotonia congenita, a rare disorder affecting skeletal muscle.

- Special attention must be paid to anesthetic management during operative procedures to prevent complications similar to those seen in patients with malignant hyperthermia.

- Our report provides a potential management plan that may be applied to $\mathrm{PC}$ patients undergoing a variety of surgical procedures and eliminates risk associated with succinylcholine and volatile anesthetics.

undergo anesthesia. ${ }^{1-2}$ Despite the known risk associated with the use of common agents such as succinylcholine and some volatile anesthetics for general anesthesia in patients with PC, there are few reports of anesthetic management for this condition. Previously reported techniques included intubation without neuromuscular relaxant for pyloromyotomy and continuous IV anesthesia with intercostal nerve block for repair of congenital nasal dysplasia using autologous costal cartilage..$^{5-6}$

Successful anesthetic induction has been reported in patients with myotonic dystrophy (a similar disorder), using rocuronium, a nondepolarizing muscle relaxant. To the authors knowledge, there are no reports of anesthetic management using rocuronium for paralysis in patients with PC.? Thus, the present report describes successful management of anesthesia in a patient undergoing laparoscopic cholecystectomy, a common surgical procedure, using non-depolarizing rocuronium for muscle paralysis, propofol and ketamine for induction, and propofol infusion/nitrous oxide for general anesthesia

BS, Medical Student. Mercer University School of Medicine, Georgia, United States

2 MD. Anesthesiologist. Anesthesia Associates of Columbus. Emory-St. Francis Hospital in Columbus, Georgia, United States.

About the Author: Analise McGreal is currently a fourth year medical student at Mercer University School of Medicine in Columbus, GA, USA. She is a member of the Alpha Omega Alpha Honor Medical Society.

Correspondence:

Analise McGreal

Address: 1550 College St, Macon, GA 31207, United States

Email: analise.elizabeth.mcgreal@live.mercer.edu
Editor: Francisco J. Bonilla-Escobar Student Editors: Azherullah Q. Syed Copyeditor: Benjamin Liu Proofreader: Adam Urback Layout Editor: Saijad Ali
Submission: Jun 5, 2020 Revisions required: Jun 27, Oct 18, 2020 Received in revised form: Aug 9, Oct 18, 2020 Acceptance: Nov 24, 2020 Publication: Dec 14, 2020 Process: Peer-reviewed 
maintenance. Written consent and Health Insurance Portability and Accountability Act (HIPAA) authorization were obtained from the patient.

\section{The Case}

A 36-year-old Caucasian female presented to the outpatient general surgery office with complaints of several months of recurrent postprandial nausea. Upper endoscopy did not reveal an obvious etiology for nausea. Subsequent diagnostic testing, including cholescintigraphy scan, was pursued and revealed a reduced gallbladder ejection fraction, diagnostic for biliary dyskinesia. Thus, it was recommended that the patient undergo elective laparoscopic cholecystectomy for symptomatic relief. The patient underwent preand peri-operative anesthesia exam, which revealed a past medical history of PC, chronic cholecystitis, gastroesophageal reflux disease (CERD), hypertension, asthma, hypothyroidism, and anxiety. The patient had not previously undergone general anesthesia, though she was aware of the risk associated with anesthesia due to her PC.

Current medications at the time of surgery included mexiletine (150 mg every 12 hours), omeprazole (40 mg each day [qd]), montelukast (10 mg every night at bed time [qhs]), azelastine hydrochloride-fluticasone propionate (137 mcg-50 mcg nasal spray twice a day [bid]), levothyroxine (50 mcg once a day [qd]), clonazepam (0.5 mg qhs), eszopiclone ( $3 \mathrm{mg}$ as needed [prn]), and propranolol (10 mg prn). Cardiovascular and respiratory review of systems revealed no significant findings, aside from history of asthma as stated (mexiletine used for chronic pain and muscle relaxation) and the patient took nothing by mouth for over 8 hours. She had not used propranolol (for anxiety) prior to surgery.

Airway exam revealed Mallampati score of 1 , thyromental distance $>3$ fingerbreadths, and mouth opening > 3 fingerbreadths. Cardiorespiratory exam revealed a regular rate and rhythm, no murmurs, rubs, or gallops, and non-labored respirations. Pre-operative labs including complete blood count (CBC) and electrolytes were within the normal limits and pregnancy test was negative. Vital signs prior to anesthetic induction included temperature of $37^{\circ} \mathrm{C}$, heart rate of 96 beats per minute, respiratory rate of 18 breaths per minute, blood pressure of $112 / 65 \mathrm{mmHg}$, and oxygen saturation of $100 \%$ on a $2 \mathrm{~L}$ nasal cannula. To maintain normothermia, the patient was covered with heated blankets in the preoperative area and in the operating room. A Bair Hugger system was also utilized throughout the duration of the case.

Core temperature monitoring was established via the esophagus and recorded periodically. Normal sinus rhythm was confirmed with electrocardiogram (EKG). Anesthetic management began with $100 \mathrm{mcg}$ of fentanyl, $4 \mathrm{mg}$ of odansetron, and $2 \mathrm{mg}$ of midazolam for pain, nausea, and anxiety. Antibiotic prophylaxis with $900 \mathrm{mg}$ of clindamycin was given. Next, $200 \mathrm{mg}$ of propofol and $20 \mathrm{mg}$ of ketamine were used for induction. Rocuronium (35 mg) was given for non-depolarizing muscle paralysis. The trachea was smoothly intubated by video laryngoscope. This was performed by a registered nurse anesthetist under the supervision of an attending anesthesiologist. Nitrous oxide and continuous propofol infusion (10 mg/mL IV emulsion $100 \mathrm{~mL}$ at 7.59 $\mathrm{mg} / \mathrm{hr}$ ) were used for maintenance of general anesthesia for the remainder of the case.

Pre-operative and post-operative electrolytes were within normal limits. Intraoperative EKG monitoring was performed throughout the entire procedure and the patient maintained normal sinus rhythm. No indication of hyperkalemia (such as peaked $T$ waves, flattening of $P$ waves, or prolongation of the PR interval) was apparent for the entirety of EKG monitoring. Thirty minutes after anesthetic initiation, an additional 50-mcg fentanyl and $20 \mathrm{mg}$ ketamine was given for pain management and sedation. Dexamethasone $4 \mathrm{mg}$ and glycopyrrolate $0.6 \mathrm{mg}$ were given just prior to the completion of anesthesia (Table 1). The patient's vitals remained stable throughout the case, with an
Table 1. Medications Used for Anesthetic Management.

\begin{tabular}{lc}
\hline Procedure & Drug and dose/Procedure \\
Antibiotic Prophylaxis & $900 \mathrm{mg}$ clindamycin \\
Induction & $200 \mathrm{mg}$ propofol, 20 mg ketamine \\
\hline Paralytic (Non-Depolarizing) & $35 \mathrm{mg}$ rocuronium \\
Anesthetic Maintenance & $\begin{array}{r}\text { Nitrous oxide via nasal cannula, continuous propofol } \\
10 \mathrm{mg} / \mathrm{mL} \text { IV emulsion 100 mL @ } 7.5 \mathrm{mg} / \mathrm{hr}\end{array}$ \\
Intraoperative Monitoring & $\begin{array}{c}\text { Potassium checks; electrocardiogram } \\
\text { monitoring; temperature via esophagus }\end{array}$ \\
End of Sedation & 4 mg dexamethasone, 0.6 mg glycopyrrolate \\
\hline
\end{tabular}

increase in systolic blood pressure prompting further pain management at 30 minutes. Total anesthetic time was 85 minutes. The patient awoke from anesthesia without complications and was discharged home on the same day, approximately 2 hours after the case concluded. She was seen for follow up in the general surgery office one week later. She reported some mental fatigue following surgery and did experience a delayed return to work as a result. She otherwise recovered well without complications.

\section{Discussion}

The present case describes successful general anesthetic management in a patient with PC using propofol and ketamine for induction andnondepolarizing rocuronium for muscle paralysis, with continuous nitrous oxide and IV propofol infusion for anesthesia maintenance. The patient remained stable throughout the case without myotonic episodes, evidence of hyperkalemia, or other complications. Given the clear contraindication to succinylcholine, this suggests that the use of nondepolarizing agents during intubation is a viable alternative strategy in patients with PC when general anesthesia is required. Moreover, it is known that use of volatile anesthetics for maintenance anesthesia poses a risk for volatile anesthetics for maintenance anesthesia poses a risk for patients with malignant hyperthermia. There are minimal reports regarding the use of volatile anesthetics in patients with $P C$, so, we opted to not use sevoflurane for maintenance in our patient to avoid unnecessary risk.

Regardless of anesthetic strategy used, hypothermia and hyperkalemia are the two biggest perioperative risks for a myotonic episode and should be monitored accordingly with a temperature probe, EKG, and electrolyte panels. Unfortunately, there is little evidence available to date regarding agents that could potentially reverse an acute myotonic episode, should one occur. There is only one reported case study in the literature that describes an acute reversal of a myotonic episode in PC during surgery. ${ }^{8}$ The patient was a 40 -year-old female who did not know that she had PC and was given succinylcholine during induction. Immediately, she was noted to have rigidity of her upper extremities, neck, and masseter muscles to the extent that her mouth could not be opened for intubation. Mask ventilation was performed and a $100 \mathrm{mg}$ IV propofol bolus was given and within 4-5 minutes her myotonia receded allowing for intubation. The surgery was then performed without complications.

Additional research has suggested that the successful reversal may be secondary to the ability of propofol to block not only wild-type sodium channels, but also mutant sodium channels in a patient with PC. ${ }^{9}$ Moreover, Matsumoto et al., reported a case that suggested the class 1B anti-arrhythmic drug mexiletine could potentially be used as a reversal agent due to its sodium channel inhibition.? If considered from a purely mechanistic view, this is logical since PC is caused by a derangement in sodium deactivation and mexiletine blocks sodium channels. However, the cited randomized control trial is somewhat limited because it reports that mexiletine is superior to placebo in reducing stiffness symptoms over a four-week period, not reversing an acute myotonic episode immediately. ${ }^{10}$ Interestingly, our patient was 
taking mexiletine at the time of surgery for chronic pain and muscle rigidity. Although it is unclear if this lowered her risk of myotonia intraoperatively, this could represent an important limitation of the present report. There were no indications for holding this medication preoperatively, and, given the beneficial effect of mexiletine in PC, doing so may not have been in the patient's best interest. It is therefore unknown, however, whether paralysis with the non- depolarizing agent rocuronium, as described in this report, would have been successful without the patient's prior prescription of mexiletine. Therefore, additional research is needed to examine anesthetic management with rocuronium in patients with PC who are not taking mexiletine. Additional areas of focus may also include whether starting mexiletine in the pre-operative period (i.e., one month prior to surgery) in patients with $P C$ shows benefit in reducing intra-operative complications for patients undergoing elective procedures.

In conclusion, our report describes successful anesthetic management using non-depolarizing muscle relaxant, rocuronium, bolus propofol, ketamine, and continuous IV propofol with nitrous oxide in a patient with PC. This provides a potential management plan that may be applied to PC patients undergoing a variety of surgical procedures and eliminates risk associated with succinylcholine and possibly volatile anesthetics. Additionally, the use of rocuronium as a muscle relaxant may allow easier and more efficient intubations in these patients,

\section{References}

1. Finsterer J. Primary periodic paralyses. Acta Neurol Scand. 2008: 117: 145-158.

2. Schieren M, Defosse J, Böhmer A, Wappler F, Gerbershagen MU. Anaesthetic management of patients with myopathies. Eur J Anaesthesiol. 2017 Oct;34(10): 641-9.

3. Parness J, Bandschapp 0, Girard T. The myotonias susceptibility to malignant hyperthermia. Anesth Analg. 2009 0ct;109(4);1054-64.

4. Bandchapp 0, laizzo PA. Pathophysiologic and anesthetic considerations for patients with myotonia congenita or periodic paralyses. Paediatr Anaesth. 2013 Sep;23(9):824-33.

5. Ay B, Gerçek A, Doğan VI, Kiyan G, Göğüş YF. Pyloromyotomy in a patient with paramyotonia congenita. Anesth Analg. 2004 Jan;98(1):68-9.

6. Matsumoto N, Nishimoto R, Matsuoka $\mathrm{Y}$, Takeda $\mathrm{Y}$, Morimatsu H. Anesthetic management of a patient with sodium-channel myotonia: a case report. JA Clin Rep. 2019 Nov;5(1):77. reducing the risk associated with prolonged hypoxia that is occasionally observed in difficult intubations. Further research is needed to determine whether this approach is superior to previously reported techniques and should also aim to identify which agents may be effectively utilized to reverse a myotonic episode in PC patients if it occurs intra-operatively.

Figure 1. Risk of Succinylcholine Utilization for Anesthetic Induction in Paramyotonia Congenita.

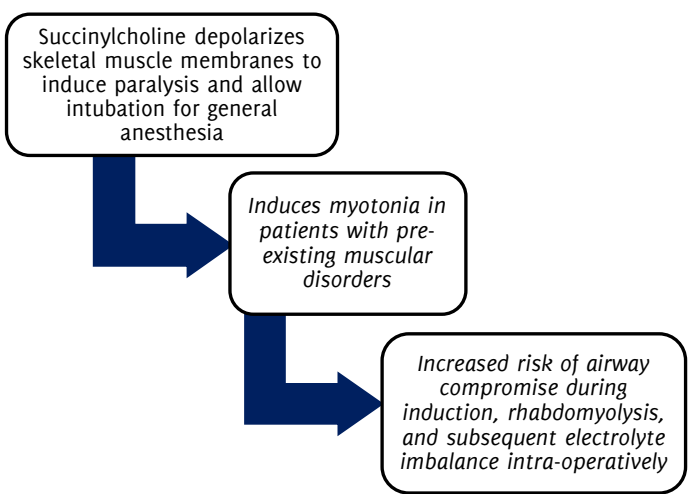

7. Vevckemans F, Scholtes JL. Myotonic dystrophies type 1 and 2: anesthetic care. Paediatr Anaesth. 2013 Sep;23(9):794-803.

8. Elsharydah A, Kaminski AC. Propofol reduces succinylcholine-induced muscle rigidity in a patient with paramyotonia congenita. Anesth Essays Res 2017 JanMar;11(1):273-4.

9. Haeseler G, Stormer M, Bufler J, Dengler R, Hecker $H$, Piepenbrock $S$, et al Propofol blocks human skeletal muscle sodium channels in a voltage-dependent manner. Anesth Analg. 2001 May;92(5):1192-8.

10. Statland JM, Bundy BN, Wang Y, Rayan DR, Trivedi JR, Sansone VA, et al Mexiletine for symptoms and signs of myotonia in nondystrophic myotonia: a randomized controlled trial. JAMA. 2012 0ct 3;308(13):1357-65.

\section{Acknowledgments}

We thank James Majors, MD, Attending Physician, Clerkship Director, Department of Surgery, Mercer University School of Medicine, Columbus, GA, USA for his surgical teaching during this patient case and for his support of this report.

\section{Conflict of Interest Statement at Funding}

The Authors have no funding, financial relationships or conflicts of interest to disclose.

\section{Author Contributions}

Conceptualization: AM, DS, AD. Data Curation, Investigation, Writing - Original Draft Preparation: AM, DS. Supervision: AM, AD. Writing - Review $九$ Editing: AM, DS, AD.

Cite as:

McGreal A, Slagle D, Dickens A. Case Report: General Anesthetic Management for Laparoscopic Cholecystectomy in Paramyotonia Congenita. Int J Med Students. 2020 Sep-Dec; 8(3):288-90.

This work is licensed under a Creative Commons Attribution 4.0 International License 